S.D. Ryder, D.J. Pisano, M.A. Walker, and K.C. Freeman, eds.

\title{
Rotating Halos and Heavy Disks: The Case of NGC 2915
}

\author{
Frédéric S. Masset \\ SAp, CE-Saclay, 91191 Gif/Yvette Cedex, France \\ Martin Bureau \\ Columbia Astrophysics Laboratory, 550 West 120th Street, 1027 Pupin \\ Hall, MC 5247, New York, NY 10027, USA
}

\begin{abstract}
NGC 2915 is a blue compact dwarf galaxy embedded in an extended, low surface brightness HI disk with a bar and two-armed spiral structure. Common mechanisms are unable to explain those patterns and disk dark matter or a rotating triaxial dark halo were proposed as alternatives. Hydrodynamical simulations were run for each case and compared to observations using customized column density and kinematic constraints. The spiral structure can be accounted for by an unseen bar or triaxial halo, but the large bar mass or halo pattern frequency required make it unlikely that the spiral is driven by an external perturber. In particular, the spin parameter lambda is much higher than predicted by current CDM structure formation scenarios. Massive disk models show that when the gas surface density is scaled up by a factor of about 10, the disk develops a spiral structure matching the observed one in perturbed density as well as velocity. This suggests that the disk of NGC 2915 contains much more mass than is visible, tightly linked to the neutral hydrogen. A classic (quasi-)spherical halo is nevertheless still required, as increasing the disk mass further to fit the circular velocity curve would make the disk violently unstable
\end{abstract}

\section{Introduction and observational properties}

NGC 2915 is an isolated blue compact dwarf galaxy at a distance $D=5.3 \pm$ 1.6 Mpc (Meurer et al. 1994). In the optical, it displays a high surface brightness blue core and a red diffuse population. The core is the locus of high mass star formation and possesses a high excitation, low metallicity HII region spectrum; the red population has an exponential profile with a low extrapolated central surface brightness. The radio properties of NGC 2915 are rather extreme, with an $\mathrm{HI}$ disk extending to 22 radial scalelengths in the $B$-band (Meurer et al. 1996, hereafter MCBF). This disk displays a short central bar overlapping the optical emission and a well-developed outer two-arm spiral extending to its edge. The signature of an oval distortion is also present in the velocity field. Standard modeling of the derived circular velocity curve yields a blue mass-to-light ratio $\mathcal{M}_{T} / L_{B} \gtrsim 65$ at the last measured point $\left(\mathcal{M}_{\text {dark }} / \mathcal{M}_{\text {luminous }} \approx 19\right)$, making NGC 2915 one of the darkest disk galaxies known. The core of the dark matter 

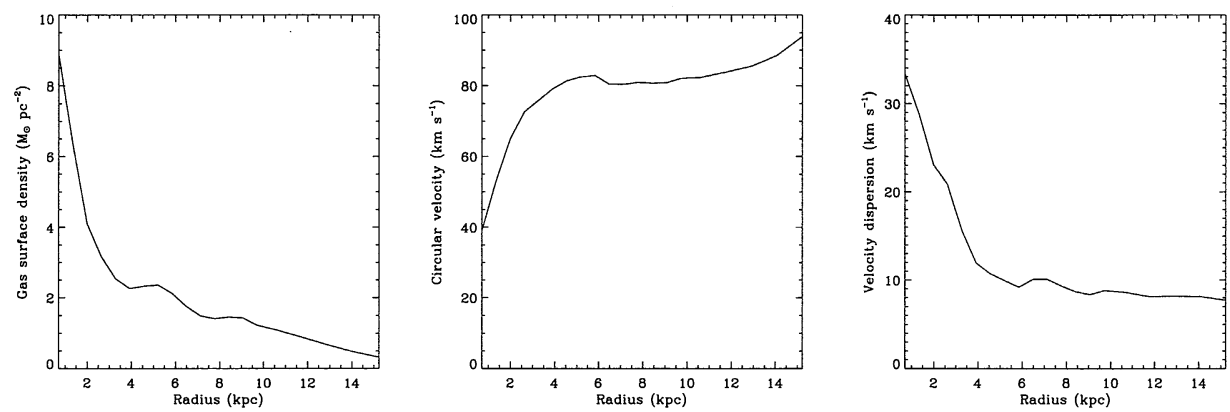

Figure 1. Azimuthally-averaged radial profiles of NGC 2915. Left: Gas surface density $\Sigma_{g}$. Center: Circular velocity $V_{c}$ (rotation curve corrected for asymmetric drift). Right: HI velocity dispersion $\sigma_{v}$. The profiles are based on uniformly-weighted data (25" beam) for $r \lesssim 10 \mathrm{kpc}$ and naturally-weighted data $\left(45^{\prime \prime}\right.$ beam) at larger radii.

halo also appears unusually dense and compact. In fact, the stellar content can be neglected at all radii without greatly affecting the fit. The optical properties of NGC 2915, discussed in more detail by Meurer et al. (1994), are thus unimportant for this work. We will rather focus on its unique HI properties, described more fully in MCBF. The circular velocity (corrected for asymmetric drift) and HI velocity dispersion profiles of NGC 2915 are shown in Figure 1. The rotation curve rises up to $R=5 \mathrm{kpc}$, where it reaches a plateau at $V_{c} \approx 80 \mathrm{~km} \mathrm{~s}^{-1}$. We note that beam smearing effects tend to overestimate the velocity dispersion in the central regions, and that the circular velocity may be underestimated. The Toomre $Q$ parameter that can be estimated from this data set has a high value everywhere in the disk, with a minimum of about 6 at $r \approx 6 \mathrm{kpc}$.

Bureau et al. (1999) discussed the origin of the HI bar and spiral pattern in NGC 2915. They argued for a common, slow pattern speed $\Omega_{p}=$ $8.0 \pm 2.4 \mathrm{~km} \mathrm{~s}^{-1} \mathrm{kpc}^{-1}$, yielding a corotation radius to bar semi-length ratio $r_{\mathrm{cr}} / r_{b} \gtrsim 1.7\left(r_{b}=180^{\prime \prime}=4.6 \mathrm{kpc}\right)$. The low (luminous) disk surface density and correspondingly high $Q$ make it unlikely that SWING amplification (Toomre 1981) can explain the spiral pattern. Bureau et al. (1999) proposed two alternatives: i) the HI disk is embedded in a massive and extended triaxial dark halo with a rotating figure, forcing the bar and spiral pattern at a certain frequency, or ii) (some) dark matter is distributed in a disk closely following the HI distribution, rendering the disk gravitationally unstable.

The purpose of this work is to test whether either (or both) of these mechanisms is valid. The rotating halo or bar is modeled by external forcing from a rotating triaxial potential, and the massive disk is modeled by simply scaling up the gaseous surface density of the disk. We constrain the models by running a simple 2D hydrodynamic code, exploring the available parameter space, and comparing with observations. We first present below the relevant parameters for both sets of models, describe suitable observational constraints, and provide a brief description of the code. The results for the external perturber models are then presented and discussed in Section 4, while those for the massive disk models are described in Section 5. 


\section{Run selection with customized observational constraints}

It is important to clearly identify the best constraints provided by the observations and the relevant model parameters one can adjust to match them. The basic constraints are provided by the successive moments of the observed data cube (i.e. the column density, mean velocity, and velocity dispersion fields), but those are not directly used. We rather prefer a custom-designed constraint based on the $m=3$ component of the deprojected line-of-sight velocity, which allows to tune the free parameters in a simple manner (see Masset \& Bureau 2003 for details). For an externally-forced disk, the free parameters are the perturber's pattern frequency and the perturber's potential amplitude (which simply scales with the bar mass for a bar and the halo triaxiality for a rotating triaxial halo). In the case of a massive disk, the only free parameter is the surface density scaling coefficient. The latter implicitly assumes that if the disk of NGC 2915 owes its spiral structure to dark material tightly linked to the HI, then the mass ratio of this material to the $\mathrm{HI}$ is independent of position. This constraint is a strong one, but it is preferable to keep the dimensionality of the parameter space as low as possible in order to get meaningful constraints.

\section{Description of the hydrodynamic code}

The hydrodynamic code used is a simple 2D Eulerian finite difference code on a polar mesh. It is described in full detail in Masset \& Bureau (2003) and references therein. Self-gravity is taken into account in all simulations but the stellar content of NGC 2915 is neglected. The disk potential is evaluated through fast Fourier transforms. The velocity dispersion is directly implemented in the code as the sound speed, which is constant in time and depends only on the distance to the center. This ensures that the disks modeled have at each instant in time a velocity dispersion profile as observed in NGC 2915.

\section{External perturber models}

\subsection{Bar forcing runs}

Figure 2 shows the best match results for a series of bar forcing runs. The pattern frequencies shown range from 5.0 to $7.5 \mathrm{~km} \mathrm{~s}^{-1} \mathrm{kpc}^{-1}$ and the spatial resolution is $N_{r} \times N_{\theta}=100 \times 250$ for all runs, with an inner grid boundary $R_{\min }=300 \mathrm{pc}$ and an outer boundary $R_{\max }=25000 \mathrm{pc}$, further than the edge of the observed HI disk. The adopted bar axis ratio $b / a=0.5$ and the isophote shape index $n=3$ are typical literature values.

We note that the bar position angle is not adjusted in the synthetic maps; it is imposed by the best match observer's azimuth $\varphi$ yielded by our fitting procedure. The fact that the modeled and observed bar position angles should coincide provides an additional constraint to the models. The bars inferred from these runs must be dark. Indeed, the maximal mass one can assign to the optical component of NGC 2915 is $M_{*} \approx 7.4 \times 10^{8} M_{\odot}$ while the HI mass in the central bar is about $5 \times 10^{7} M_{\odot}$, to be compared to the bar masses needed in our runs which are of order $4-7 \times 10^{9} M_{\odot}$. 

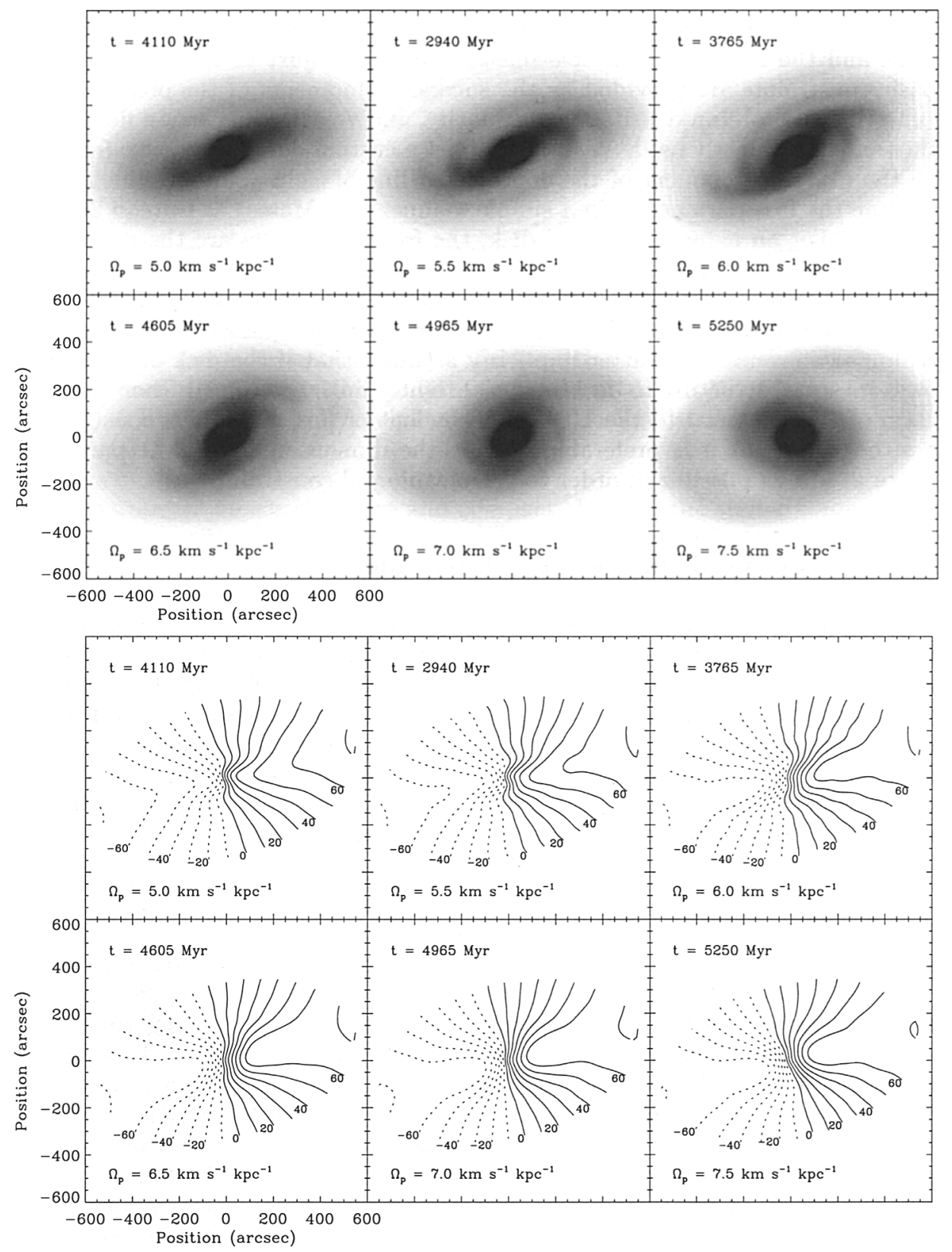

Figure 2. Synthetic column density and line-of-sight velocity maps for our best bar forcing runs. The time and pattern frequency are indicated in each panel. Convolution by a $45^{\prime \prime}$ beam has been applied. 


\subsection{Triaxial halo runs}

We have also performed a series of runs in which the disk response is excited by a rotating triaxial halo of the same set of pattern frequencies as the bar forcing runs discussed above. The axis ratios obtained vary between 0.83 and 0.88 for pattern frequencies between 4.5 and $9.5 \mathrm{~km} \mathrm{~s}^{-1} \mathrm{kpc}^{-1}$. From these runs, we estimate plausible values of $\Omega_{p}$ to be at most $8 \mathrm{~km} \mathrm{~s}^{-1} \mathrm{kpc}^{-1}$, consistent with the bar forcing results.

This pattern frequency is however much higher (by one order of magnitude) than those expected for the figure rotation of triaxial dark halos, as suggested by angular momentum statistics in $\Lambda$ CDM simulations (assuming that streaming motions internal to the halo contribute negligibly to its angular momentum). It is therefore unlikely that NGC 2915 owes its spiral structure to forcing by a triaxial halo. We note however that the collision of two halos can produce a fast precessing halo, which could in turn trigger spiral structures as observed in NGC 2915 (Bekki et al. 2004, these proceedings).

\section{Heavy disk runs}

The heavy disk simulations use the same code as the external perturber simulations, with the exception that we filter out small scale potential components. We also scale up the observed gaseous surface density by a uniform scalar $\lambda_{\Sigma} \geq 1$. As the spiral structure driver is now an instability internal to the disk, it is no longer necessary to invoke any external triaxiality and the resulting disk is embedded in a spherical halo whose radial density profile is constructed for each $\lambda_{\Sigma}$ to match the observed circular velocity. We use the observed velocity dispersion profile directly and disregard any possible beam smearing or other effects. The only free parameter is thus the dimensionless scalar $\lambda_{\Sigma}$, which we vary between 1 and 6 , at which value Toomre's $Q$ parameter is approximately unity at $r=6.5$ and $9 \mathrm{kpc}$ and the disk becomes globally unstable to axisymmetric modes. The goal is to find the range of $\lambda_{\Sigma}$ where a spiral instability develops, leading to the formation of a grand-design spiral structure similar to the one observed and, if possible, to further constrain the value of $\lambda_{\Sigma}$ so that the amplitude of the spiral also matches. We again used the observed $m=3$ amplitude component of the deprojected velocity field as our main constraint, and the best match is obtained for $\lambda_{\Sigma}=4.93$. Figure 3 shows the corresponding density and velocity maps. We stress that the observer's azimuth in the three panels of that figure is the same, and that the position angle of the spiral structure is not adjusted. We thus see that it is possible to account for most of the large-scale structure in the disk of NGC 2915 by simply uniformly scaling up the observed gas surface density.

In order to provide a reliable estimate of the scaled-up mass in the disk of NGC 2915, one has to estimate finite thickness effects since these provide a cut-off of the $m=2$ potential component arising from the spiral structure (see Masset \& Bureau 2003 for details). Those corrections lead to a disk surface density scaling factor of about 10 for NGC 2915, in good agreement with those derived for a variety of spiral galaxies (where typical values are $6-10$, with a tail at the high end). We note however that the above values are normally determined by attempting to fit the circular velocity curve only (with a scaled-up $\mathrm{HI}+\mathrm{He}$ disk and a stellar component with free $\mathcal{M} / L$ ), while our goals here differ 

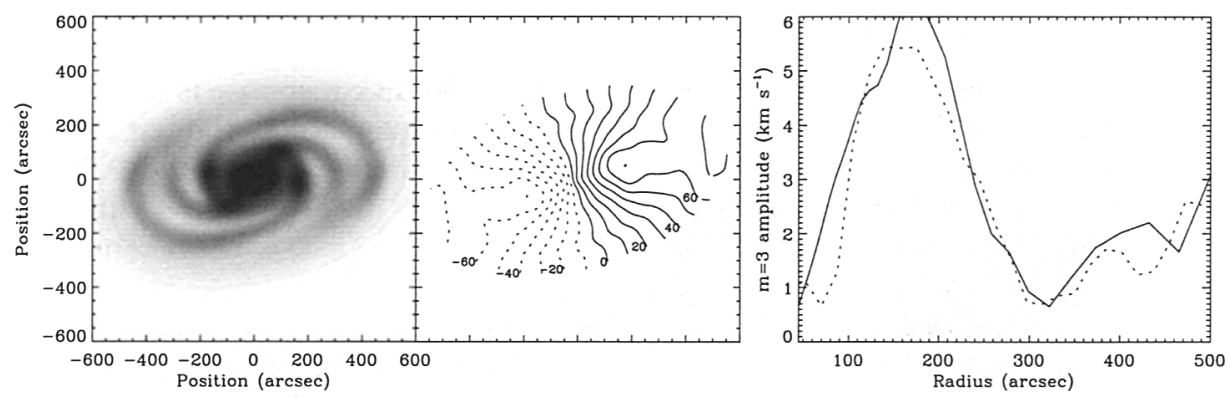

Figure 3. Heavy disk run best match. The left panel shows the beam convolved simulated HI column density (properly scaled), the middle panel the line-of-sight velocity field, and the right panel the radial dependence of the $m=3$ component of the deprojected line-of-sight velocity (solid line: model, dotted line: observations).

and we allow for an extra dark halo. Such circular velocity curve fitting would yield a scaling factor of about 25 for NGC 2915 , but it would poorly reproduce the total circular velocity. In fact, because this scaling factor is much higher than that obtained from the requirement of spiral structure sustainability only, the resulting disk would be violently unstable to axisymmetric perturbations. This strongly argues for the presence of a classical (i.e. spherical or quasi-spherical) dark matter halo in NGC 2915 in addition to any realistic massive disk.

\section{References}

Bureau, M., Freeman, K. C., Pfitzner, D. W., \& Meurer, G. R. 1999, AJ, 118, 2158

Masset, F. S., \& Bureau, M. 2003, ApJ, 586, 152

Meurer, G. R., Carignan, C., Beaulieu, S. F., \& Freeman, K. C. 1996, AJ, 111, 1551 (MCBF)

Meurer, G. R., Mackie, G., \& Carignan, C. 1994, AJ, 107, 2021

Toomre, A. 1981, in Structure and Evolution of Normal Galaxies, eds. S. M. Fall \& D. Lynden-Bell (Cambridge: Cambridge University Press), 111 